\title{
Metabolismo del colesterol su regulación a nivel hepático e intestinal
}

\author{
Por M. T. Molina*, C. M. Vázquez y V. Ruíz Gutiérrez ${ }^{\star *}$ \\ *Facultad de Farmacia. Universidad de Sevilla. \\ ** Instituto de la Grasa y sus Derivados. Avda. Padre García Tejero, 4. 41012 - SEVILLA.
}

\section{RESUMEN}

Metabolismo del colesterol. Su regulación a nivel hepático e intestinal.

Aunque todas la células del organismo tienen capacidad para sintetizar colesterol, la mayor parte de la síntesis de éste, que da lugar a lo que se conoce como colesterol endógeno, se realiza en el hígado. El hepatocito tiene además capacidad de captar colesterol de las lipoproteínas circulantes, y a la vez de excretarlo formando parte de nuevas lipoproteinas de origen hepático o transformado en ácidos biliares. El colesterol de origen extrahepático procede principalmente de la mucosa intestinal. Aquí se realiza la absorción del colesterol de la dieta (colesterol exógeno), la biosíntesis de nuevo colesterol y la esterificación para ser almacenado en la célula o secretado a sangre en las lipoproteínas de origen intestinal.

A nivel celular, la importancia del colesterol radica en que forma parte de la mayoria de las estructuras membranosas de todas las células del organismo.

En este artículo se revisan algunos de estos aspectos del metabolismo del colesterol, y se analiza la influencia de la composición lipídica de un tipo de membranas celulares, las membranas microsomales, en la actividad de los enzimas reguladores del metabolismo de colesterol.

PALABRAS-CLAVE: Actividad enzimática - Colesterol (metabolismo) - Composición lipídica - Información (artículo) Membrana.

\section{SUMMARY}

Cholesterol metabolism. Its regulation at the hepatic and intestinal level.

Although all the cells in the body are able to form cholesterol, most part of this synthesis, leading to which is called endogenous cholesterol, occurs in the liver. Hepatocytes can also obtain cholesterol from the plasma lipoproteins. At the same time, cholesterol is either secreted from the liver in new plasma lipoproteins or transformed in bile acids. The extrahepatic cholesterol is mainly produced in the intestinal mucosa. In the site, it takes place the absorption of cholesterol from the diet

Abreviaturas: HMG-CoA. 3-hidroxi-3-metilglutaril coenzima A ACAT. acil-coenzima A colesterol-aciltransferasa. (exogenous cholesterol), along with the biosynthesis of new cholesterol and the esterification of the molecule to be stored in the cell or secreted as plasma lipoproteins.

At the cellular level, the importance of cholesterol comes from the fact that many of the membranous structures of all cells are partially composed of these substance.

In this article some of these aspects of the cholesterol metabolism are reviewed. We also describe the influence of lipid composition of microsomal membranes on the activity of cholesterol metabolism regulating enzymes.

KEY-WORDS: Cholesterol (metabolism) - Enzymatic activity - Information (paper) - Lipid composition - Membrane.

\section{INTRODUCCION}

El colesterol fue descubierto en 1770 por el francés Poulletiere de la Salle, quien lo aisló de los cálculos biliares, por lo que. en un principio se denominó colestiramina (grasa biliar), y luego pasó a llamarse colesterina (chole=bilis y stereos=sólido). Finalmente se denominó colesterol cuando se comprobó la presencia de un $\mathrm{OH}^{-}$en su molécula. En 1843, Vogel detectó la presencia de colesterol en placas ateromatosas. Desde entonces, los análisis clínicos y las observaciones histológicas establecieron que la aterosclerosis y sus complicaciones cardíacas están ligadas al metabolismo anormal de los lípidos, asociándose el colesterol con enfermedades cardiovasculares. Pero no podemos olvidar que el colesterol es un elemento esencial para la mayoría de las células del organismo, siendo la regulación de su metabolismo de vital importancia para un buen funcionamiento de las mismas. En el hepatocito, esta regulación resulta de un equilibrio entre, la llegada de colesterol vía lipoproteínas plasmáticas, la síntesis de novo de colesterol en la propia célula y la salida de éste en forma de lipoproteínas y como componente de la bilis. A su vez, el colesterol puede ser almacenado en la célula en forma de éster o formar parte de las membranas celulares como colesterol libre (ver Esquema 1). 


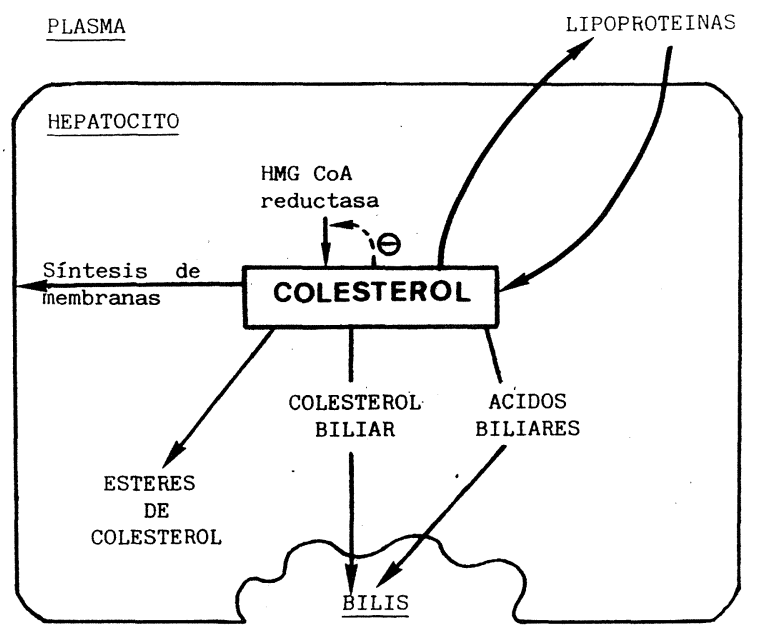

Esquema 1

Metabolismo hepático del colesterol. El hepatocito obtiene el colesterol de las lipoproteinas circulantes o mediante su biosíntesis. A su vez, la célula emplea el colesterol para la sintesis de membranas, secreción de ácidos biliares y formación de lipoproteinas. El exceso de colesterol se almacena como colesterol esterificado. La enzima HMG-CoA reductasa regula la boisíntesis de colesterol mediante retroinhibición por el sustrato formado (tomado de Packard y Shepherd, 1982)

\section{ORIGEN DEL COLESTEROL HEPATICO}

Para analizar la procedencia del colesterol hepático vamos a dividir esta sección en dos apartados, el primero biosíntesis y segundo, lipoproteínas plasmáticas como fuentes de colesterol.

\section{Biosíntesis del colesterol. Actividad HMG-CoA re- ductasa}

La síntesis de colesterol en el hepatocito se produce a partir de acetil-CoA, mediante una cadena de reacciones de condensación, reducción e hidroxilación (1). En numerosas especies animales, incluida el hombre, la reacción que limita esta síntesis es la conversión de HMG-CoA a mevalonato, catalizada por la enzima HMG-CoA reductasa (E.C.1.1.1.34). Esta enzima utilizada NADPH como reductor y su pH óptimo es de 7.

Se ha encontrado actividad reductasa en muchos tejidos de mamíferos, y siempre está asociada al retículo endoplásmico de la célula. En el hígado e intestino se han detectado las mayores cantidades de este enzima, de forma que de $2 / 3$ a $3 / 4$ partes del colesterol total del organismo se sintetiza en estos órganos (2) (3).

La actividad reductasa sufre cambios apreciables durante el desarrollo del feto y en el recién nacido, hasta alcanzar los valores basales en el adulto, acompañados de idénticas fluctuaciones en la síntesis de colesterol (3). Esta enzima presenta, además, un ritmo circadiano, con un máximo de actividad durante la noche, tanto en ratas (4) como en pollos (5), detectándose este ritmo ya en las primeras semanas de vida Gill et al. (2) han postulado que el ritmo circadiano de la reductasa está bajo control hormonal, y su variación resulta de una alteración compleja de hormonas tales como la insulina, glucagón, corticoides, prolactina, T3, etc., cuyos niveles a su vez cambian en respuesta al tipo de alimentación.

Entre los posibles factores que podrían actuar modulando la actividad reductasa se encuentran, a) la composición lipídica de las membranas microsomales, b) proteínas citosólicas transportadoras de lípidos $y$, c) sales biliares (6) (7) (8).

En general, la mayoría de las variaciones en la actividad reductasa en distintas situaciones se deben, al menos en parte, a cambios en la cantidad de enzima (regulación a largo plazo). Shapiro y Rodwell (4) demostraron que la cicloheximida, conocido inhibidor de la síntesis de proteínas, anulaba el aumento de actividad reductasa durante el período de oscuridad. Igualmente, Rodwell et al. (9). midiendo la incorporación de Leu (H3) en reductasa purificada desmostraron que los cambios en la actividad detectada a lo largo del día se debían a un aumento en la síntesis de nueva proteína, sin alterarse su degradación. El ayuno durante 24 horas, produce una disminución de actividad en ratas sin cambios en la proporción de enzima defosforilada $(\mathrm{Ra})$ respecto a la enzima total (Rt). Por el contrario, el stress y la colestiramina aumentaron los niveles de actividad sin alterar la relación $\mathrm{Ra} / \mathrm{Rt}$ en ninguno de los casos (10).

\section{Lipoproteínas plasmáticas}

Las lipoproteínas circulantes (VLDL, LDL y HDL), constituyen otra fuente de colesterol para el hepatocito, aunque la síntesis de novo del colesterol, citada en el capítulo anterior, es cuantitativamente más importante. De todas las lipoproteínas circulantes parece ser que las lipoproteínas de alta densidad (HDL) son las que transpotan la mayor parte del colesterol en el plasma sanguíneo de la rata. Por el contrario, en el hombre son las LDL (11). En el interior de estas lipoproteínas el colesterol es transportado en dos formas, que ocupan diferentes sitios en la partícula; el colesterol libre se localiza principalmente en la superficie, junto con fosfolípidos y proteínas, mientras que el colesterol esterificado y los triglicéridos forman parte de la matriz hidrofóbica de las lipoproteínas (11).

El colesterol de las LDL es captado por la célula hepática mediante la interacción de la lipoproteína con receptores específicos de membrana de alta afinidad. Esta vía está autorregulada, de forma que cuando la 
célula tiene cubiertas sus necesidades de colesterol se suprime la síntesis de receptores de LDL. Por el contrario, ante una demanda de colesterol, la síntesis de receptores específicos para LDL se ve aumentada (1) (2).

\section{RUTAS METABOLICAS DEL COLESTEROL HE- PATICO}

El colesterol hepático de fuente endógena (síntesis) o exógena (lipoproteínas circulantes) puede sufrir los siguientes procesos:

1) ser almacenado intracelularmente como colesterol esterificado,

2) excretarse en bilis,

3) incoporarse a la síntesis de lipoproteínas y,

4) formar parte de las membranas celulares del propio hepatocito. A continuación consideraremos estas distintas rutas metabólicas (ver Esquema 1).

\section{Esterificación de colesterol. Actividad ACAT}

El colesterol puede ser almacenado en la célula como éster de ácidos grasos de cadena larga, siendo la enzima responsable de esta reacción la ACAT. Esta es una enzima microsomal, presente prácticamente en todas las células del organismo, incluido el hígado. Como sustrato utiliza el colesterol de la misma membrana microsomal y, en cuanto a los ácidos grasos, la afinidad más alta la muestra por oléico y palmítico (12) (13).

Al igual que la HMG-CoA reductasa, la ACAT hepática presenta un ritmo circadiano paralelo al de aquella (6), y su actividad está regulada por un mecanismo de fosforilación/defosforilación, pero de manera inversa, pues mientras la reductasa se inactiva por fosforilación la ACAT lo hace por defosforilación(14).

La síntesis de ésteres de colesterol en el hígado se realiza bajo control de diversos factores. Uno de ellos parece ser la biodisponibilidad de colesterol celular. Tanto in vivo (15), como in vitro (16), se ha observado una relación directa entre actividad ACAT y biodisponibilidad de colesterol.

Otro factor que parece afectar la actividad de esta enzima, es la composición lipídica de la membrana microsomal. La alteración de la relación fofolípidos/ colesterol de las membranas biológicas produce un cambio en la fluidez de las mismas, lo que da lugar a cambios en las actividades de las enzimas integradas en esas membranas (17).

También se tiene evidencia de que la composición en ácidos grasos de la membrana microsomal regula de alguna forma la actividad de ACAT. En este sentido, se ha asociado un aumento en el porcentaje de ácidos grasos poliinsaturados con un aumento en la actividad ACAT, sin alteraciones apreciables en las cantidades de colesterol en las membranas, ni en la de fosfolípidos (18). Otros autores asocian los cambios en la actividad ACAT con variaciones en las proporciones de ácidos grasos poliinsaturados de las series (n-6) y $(n-3)$ en dichas membranas (19) (20).

Por otra parte, la ACAT hepática parece estar, igualmente, bajo control de las lipoproteínas circulantes (11). Así, se ha comprobrado que una disminución de LDL circulantes da lugar a un aumento de la actividad HMG-CoA reductasa y del número de receptores de LDL, mientras que la actividad ACAT decae hasta valores mínimos. Por el contrario, ante un aumento de LDL circulantes, los niveles de colesterol intracelular se elevan hasta hacer suprimir la actividad HMG-CoA reductasa y aumentar la actividad ACAT.

Recientemente, Gavey et al. (21) han estudiado el papel de la $\mathrm{SCP}_{2}$ (proteína citosólica transportadora de esteroles) en la esterificación del colesterol por la ACAT, demostrando que, cuando al medio de incubación se agrega $\mathrm{SCP}_{2}$, la conversión del colesterol a sus ésteres se ve aumentada notablemente, y postulan que la $\mathrm{SCP}_{2}$ posiblemente transporte la molécula de colesterol hasta la zona de la membrana microsomal donde se encuentra la enzima ACAT, favoreciendo así su esterificación.

\section{Otras vías de utilización del colesterol hepático}

Una de las vías de eliminación hepática del colesterol es su secreción en bilis, bien como colesterol libre o como ácidos biliares. No está muy claro la relación existente entre la biosíntesis de colesterol y su excreción en la bilis. En el hombre se ha encontrado una relación directa entre ambos hechos. Sin embargo, esta relación parece no existir para otras especies (11).

Se sabe que el colesterol en la bilis se mantiene en solución por la acción detergente de las sales biliares, de forma que la secreción de sales biliares y colesterol parece estar acoplada. Las sales biliares se sintetizan en el hígado a partir del colesterol, por saturación e hidroxilación del mismo y posterior escisión oxidativa de su cadena lateral. Generalmente se acepta que la reacción inicial en la síntesis hepática de sales biliares es la introducción de un grupo hidroxilo en la posición $7 \mathrm{del}$ anillo de colesterol. Esta reacción está catalizada por la enzima microsomal colesterol$7 \alpha$-hidroxilasa (22) y se ha demostrado que su sustrato de elección preferente es el colesterol libre de nueva sintesis (14) (23). 
Otra vía de excreción del colesterol es mediante la formación y secreción de lipoproteínas (principalmente VLDL y HDL). Parece ser que la síntesis y secreción de VLDL, lipoproteína rica en triglicéridos, está estrechamente relacionada con la síntesis de colesterol y la actividad HMG-CoA reductasa. Así, se ha demostrado que en ratas y en el hombre la estimulación de la secreción de VLDL se acompaña de un aumento en la actividad HMG-CoA reductasa, y que la secreción hepática de VLDL exhibe el mismo ciclo circadiano que la HMG-CoA reductasa (11). Basándose en estos resultados se propuso que, el aumento en la síntesis de VLDL disminuiría el "pool" de colesterol hepático, cuya consecuencia inmediata era el aumento de la actividad HMG-CoA reductasa, mediante un mecanismo de retroalimentación negativo (11).

También, la ACAT hepática podría desempeñar un papel importante en la síntesis de lipoproteínas a partir de ésteres de colesterol, ya que la estimulación de la actividad ACAT en cultivos de hepatocitos da lugar a un aumento en la secreción de VLDL (24).

Sin embargo, la excreción de colesterol como HDL, no parece depender de cambios en el metabolismo de triglicéridos ni de actividad HMG-CoA reductasa (11).
Finalmente, el colesterol libre intracelular del hepatocito también puede ser destinado a la formación de membranas de la propia célula.

\section{METABOLISMO DE COLESTEROL EN EL INTES- TINO}

La mucosa intestinal juega un papel importante en la regulación del metabolismo del colesterol en el organismo, debido, a que: 1) en la mucosa intestinal se produce la absorción de colesterol exógeno (procedente de la dieta) y endógeno (colesterol biliar), 2) el intestino es el órgano más importante, después del hígado, para la biosíntesis de colesterol y, 3) el enterocito secreta colesterol al sistema circulatorio en forma de lipoproteínas (quilomicrones).

El "pool" de colesterol en el enterocito debe mantenerse constante para cumplir eficazmente dos funciones:

1. - - Mantener el aporte de colesterol necesario para la síntesis y diferenciación de membranas celulares, ya que la superficie mucosal se haya continuamente en renovación,

2. ${ }^{\text {- }}$ Facilitar colesterol para la incorporación a lipoproteínas durante la absorción de triglicéridos.

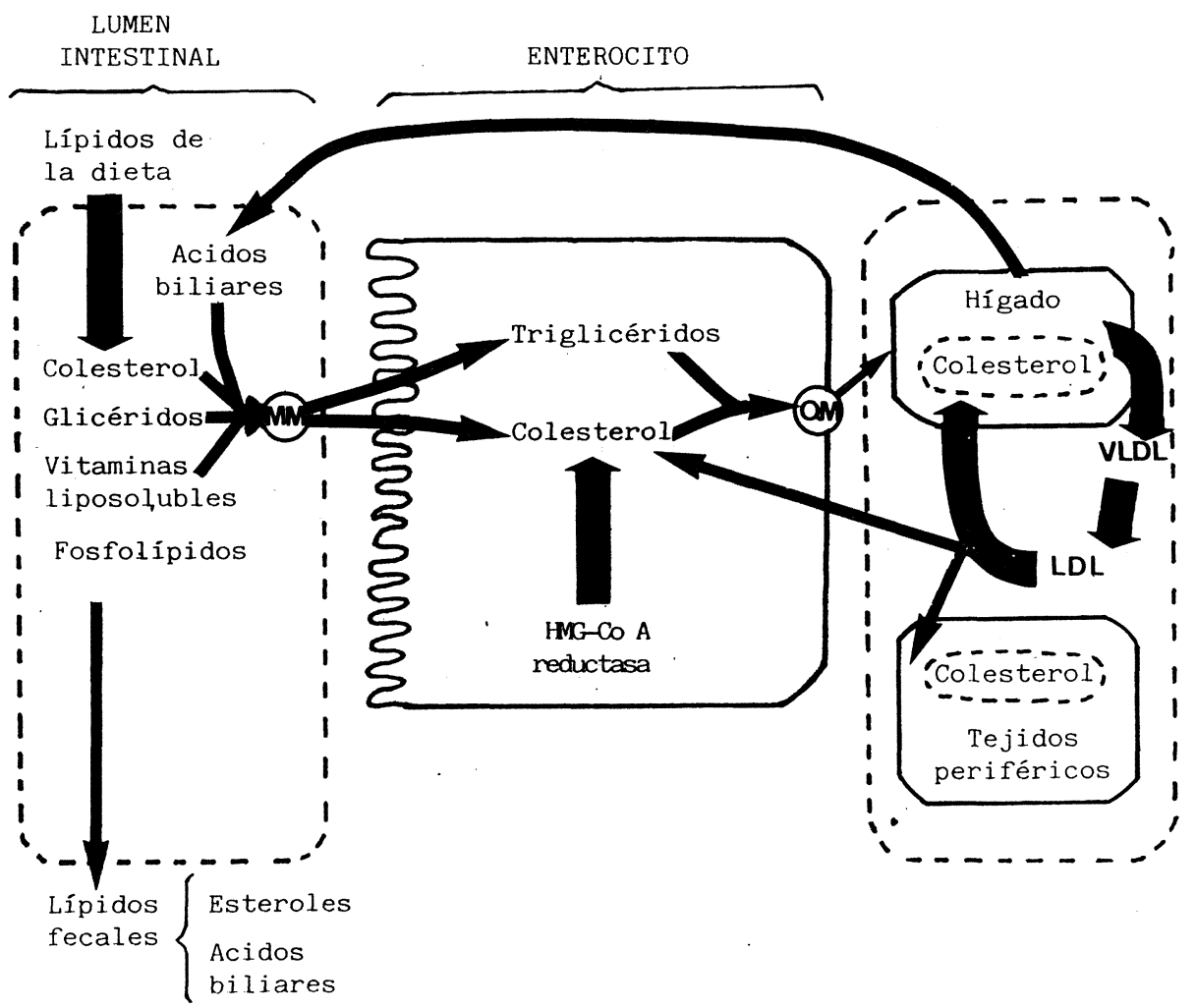

Esquema 2

Balance de colesterol a través de la célula del epitelio intestinal. Tras la digestión, los lípidos se combinan con las sales biliares en el lumen intestinal para formar las micelas mixtas (MM) que hacen posible la absorción de colesterol. Dentro de la célula intestinal triglicéridos y colesterol, además de apoproteinas específicas, forman los quilomicrones (QM), que transportan la mayoría del colesterol al hígado (tomado de Stange y Dietschy, 1985). 
La regulación del metabolismo de colesterol en el enterocito se basa en mantener en equilibrio el aporte y salida de colesterol celular, de manera análoga a lo expuesto para el hígado. En el Esquema 2 se representa el papel que desempeña la célula intestinal en el metabolismo del colesterol, así como su relación con el hígado y otros tejidos periféricos.

A continuación se revisan las fuentes de colesterol para el enterocito y la utilización de éste por la célula epitelial.

\section{Absorción intestinal de colesterol}

Una de las fuentes principales de colesterol para la célula intestinal es el colesterol procedente de la dieta o de la bilis. Durante mucho tiempo se ha estudiado el mecanismo por el cual los lípidos (colesterol) se absorben en el tracto gastrointestinal. Generalmente se acepta que el colesterol, al ser una molécula liposoluble, se absorbe desde la luz intestinal mediante un proceso de difusión pasiva, por dilución en la membrana de borde en cepillo del enterocito. El colesterol esterificado que entra en el lumen intestinal, es hidrolizado, antes de ser absorbido, por la esterasa de colesterol pancreática.

Por otra parte, para la absorción de los lípidos (colesterol) es esencial la participación de las sales biliares (25). Estas son sustancias anfipróticas, que en solución forman micelas, y estas micelas actúan englobando los monoglicéridos y ácidos grasos, junto con el colesterol manteniéndolos en suspensión (26).

Se ha demostrado en ratas, que el colesterol de la dieta no se comporta de la misma forma que el colesterol biliar, siendo este último absorbido en mayor cuantía. Por otro lado, mientras la absorción de colesterol biliar se da principalmente en el yeyuno, el procedente de la dieta se absorbe a lo largo de todo el intestino delgado, preferentemente en la segunda y tercera cuarta parte (27).

Por otra parte, Gallo-Torres et al. (28) utilizando una técnica de canulación del conducto torácico in vivo, demostraron que las distintas sales biliares presentan diferente efectividad en la absorción de colesterol. Así, el taurocolato da lugar a absorciones de colesterol de igual rango que la bilis y el glicolato y glicodeoxicolato son más efectivos que los correspondientes tauroconjugados. Además, la mayor proporción de colesterol esterificado encontrada en linfa se corresponde con la administración de trihidroxilados, con independencia de su conjugación o no. Sin embargo, todas estas observaciones sobre la relación entre la absorción de colesterol libre y esterificado y sales biliares, no se corresponden con la solubilidad in vitro del colesterol, utilizando estas mismas sales.
Thomson et al. (29) han observado que la absorción de colesterol varía según las especies, siendo en el hombre mayor que en la rata. Además, la absorción de colesterol, sufre, también, cambios a lo largo del desarrollo; así, por ejemplo, en conejos, la absorción de colesterol es mayor en animales jóvenes que en viejos, probablemente debido a la mayor permeabilidad y superficie funcional de las membranas en animales jóvenes (30). Por el contrario, en la rata la absorción in vivo de colesterol aumenta con la edad (31). Así mismo, la captación de colesterol varía con la zona del intestino estudiada, siendo más alta en yeyuno que en íleon (32) y absorbiéndose principalmente en la zona media de la vellosidad (33).

Por otro lado, la permeabilidad pasiva de la membrana intestinal al colesterol se altera en respuesta a manipulaciones en el contenido de los distintos nutrientes de la dieta (34). Una dieta rica en ácidos grasos saturados da lugar a un aumento en la absorción de colesterol a los 14 días de comenzar dicha dieta, efecto que no se observa con una dieta suplementada con ácidos grasos insaturados, aún cuando la absorción vuelve a ser normal cuando se restituye la dieta estandar (34). Por el contrario, estos cambios no se ven acompañados por variaciones en los niveles de colesterol de la membrana de borde en cepillo (35).

\section{Biosíntesis del colesterol en la mucosa intestinal}

Como ya se ha indicado, el intestino junto con el hígado son los dos órganos principales para la síntesis de colesterol en mamíferos. En la rata, el intestino contribuye con un $25 \%$ y el hígado con un $50 \%$ a la síntesis total de colesterol en el organismo y, al igual que ocurre en el hígado, la enzima reguladora de esta síntesis es la HMG-CoA reductasa (36) (37). En esta especie animal, se ha detectado actividad HMG-CoA reductasa a lo largo de todo el tracto gastrointestinal, desde el esófago al colon. Sin embargo, las mayores proporciones de síntesis de esteroles se dan en estómago, duodeno, íleon terminal y colon distal, registrándose las más bajas en el intestino delgado medio, donde tiene lugar, a su vez, la absorción de colesterol. Esta distribución puede variar con la especie (37) (38). También existe un gradiente de actividad biosintética a lo largo del eje cripta-villus (38).

De forma análoga al hígado, los cambios en la actividad de la HMG-CoA reductasa intestinal pueden deberse a alteraciones en la síntesis o degradación de la enzima, o a variaciones en la proporción de enzima fosforilada, dependiendo de que la regulación sea a largo o a corto plazo, respectivamente (39) (40). Sin embargo, según Field et al. (33) la HMG-CoA reductasa intestinal es menos susceptible que la 
hepática a la modulación por fosforilación-defosforilación. Igualmente, la reductasa intestinal presenta un ritmo circadiano, que coincide con el de la hepática, aunque de menor amplitud (38).

La actividad de la HMG-CoA reductasa intestinal, y como consecuencia la síntesis de colesterol en las células de la mucosa intestinal, viene determinada por el contenido celular de colesterol que depende de: 1) la velocidad de absorción del colesterol desde la luz intestinal (ya mencionada), 2) la velocidad de captación de lipoproteínas circulantes por la membrana basal de la célula epitelial $y, 3$ ) las necesidades de colesterol que tenga la célula para formar quilomicrones y su propia estructura (ver Esquema 3).

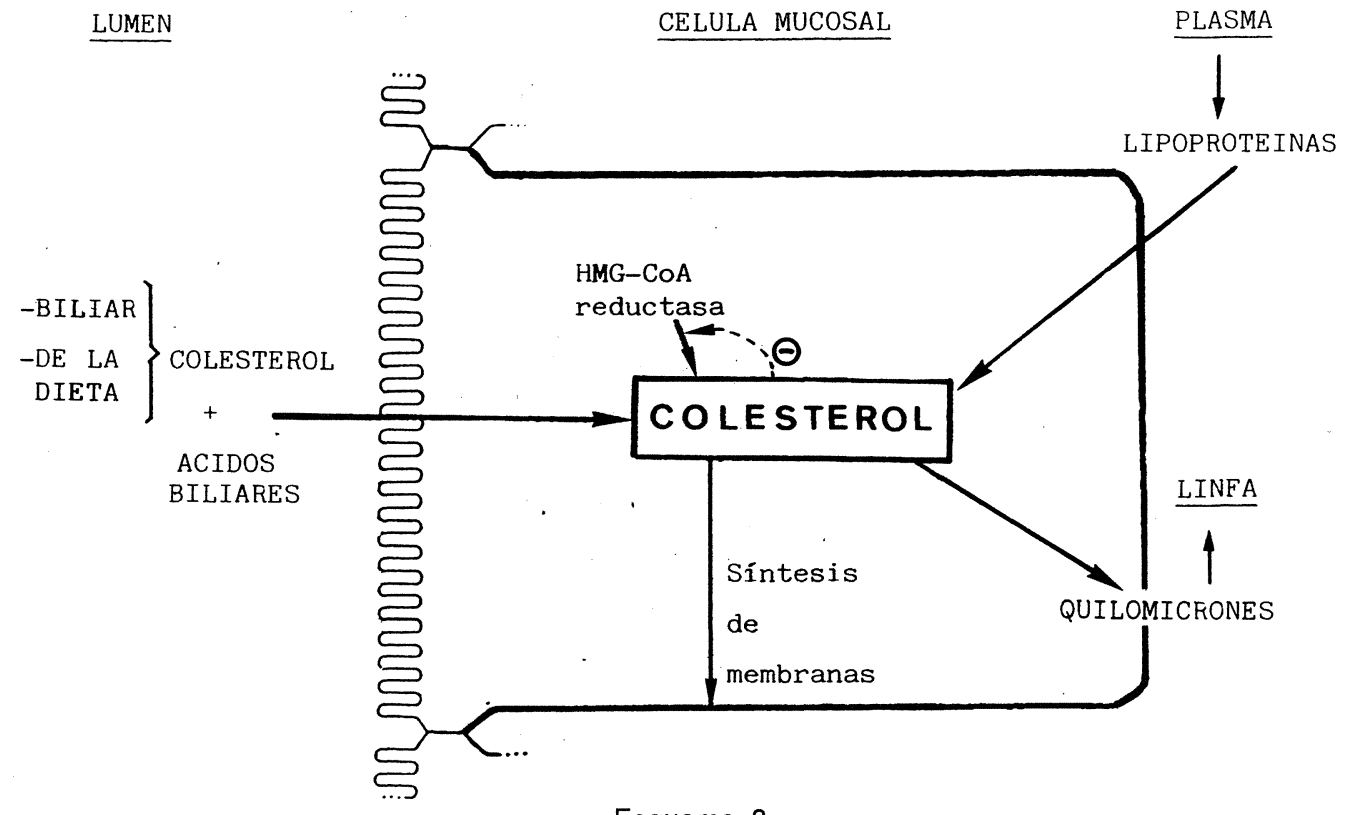

Esquema 3

Metabolismo del colesterol intestinal. La célula de la mucosa intestinal obtiene el colesterol a partir de tres fuentes: del lumen intestinal mediante su absorción, por síntesis directa de éste o a partir de las lipoproteínas circulantes. Parte del colesterol se utiliza para la síntesis de membranas, y otra parte sale a la circulación linfática en forma de quilomicrones. La HMG-CoA reductasa regula la biosíntesis de colesterol mediante retroinhibición por el sustrato (tomado de Packard y Shepherd, 1982).

En un principio se propuso que la biosíntesis de colesterol en el intestino dependía del "pool" de sales biliares (41). Sin embargo, estudios posteriores sugieren que los cambios en el "pool" de sales biliares alteran la síntesis de colesterol de forma secundaria, es decir, afectando la absorción intestinal de colesterol, siendo el colesterol molecular el responsable de su propia síntesis (38) (42). En este sentido, se comprobó que algunos bloqueantes de la absorción de colesterol (surformer, diosgenina) aumentaban la colesterogénesis a lo largo del eje cripta-villus (38).

Otra fuente de colesterol para la célula epitelial es la captación de lipoproteínas circulantes (LDL) por la membrana basolateral. Recientemente se ha demostrado que en la rata y en el hamster, el intestino es el segundo lugar más importante para la degradación de LDL después del hígado. El 50-60\% de la captación de LDL por el enterocito depende de la interacción de la lipoproteína con receptores específicos, siendo la captación no dependiente de receptores más importante en hígado y glándulas endocrinas (38). Se ha comprobado, que inhibidores de la secreción hepática de lipoproteínas producen un aumento de la actividad HMG-CoA reductasa en el intestino, que revierte con la administración intravenosa de LDL. De igual manera, en cultivos de células epiteliales se ha comprobado que las LDL suprimen la actividad reductasa intestinal (43). Estos estudios ponen de manifiesto que esta actividad reductasa en la célula intestinal viene regulada por la captación de lipoproteínas circulantes.

Además, pueden afectar a la actividad reductasa los cambios en las necesidades de colesterol que presente la célula para formar quilomicrones o para la propia estructura de sus membranas. Stange et. al (42), comprobaron que el aumento en la ingestión de triglicéridos daba lugar a un aumento en la síntesis de colesterol, lo que sugiere que el colesterol sintetizado de novo podría ser utilizado para la producción de quilomicrones (38).

La mayor síntesis de colesterol encontrada en las células de la cripta respecto a la de las células superiores de la vellosidad, sugieren que la mayor parte del colesterol sintetizado es incorporado en las membranas de las nuevas células, contribuyendo así a la formación y maduración del epitelio intestinal. 
Por último, al igual que en el hígado, la actividad HMG-CoA reductasa intestinal puede depender de cambios en la fluidez de la membrana, y aunque existe controversia en este tema, Field et al (44) sugieren que la regulación de la enzima intestinal se relaciona con el grado de saturación de los ácidos grasos de la membrana microsomal.

\section{Esterificación del colesterol en el intestino.}

El colesterol se absorbe desde el lumen intestinal como colesterol libre. Sin embargo, la mayor parte del colesterol encontrado en linfa está esterificado. Por esta razón, algunos autores sugieren que la esterificación de colesterol es uno de los pasos limitantes para la absorción intestinal de este nutriente (38).

Se han descrito tres sistemas de esterificación del colesterol: ACAT intestinal, colesterol esterasa (CE) y colesterol éster-sintetasa (CES) (38).

La ACAT intestinal fue descrita por Haugen y Norum en 1976 y se localiza en la membrana microsomal del enterocito, catalizando la misma reacción que la ACAT hepática. Tiene un $\mathrm{pH}$ óptimo de reacción de 7.2 y requiere ácidos grasos libres y un sistema regenerador de ATP o acil-CoA. El orden de preferencia por los sustratos es oleil>palmitoil>estearil>linoleil-CoA de manera análoga a la ACAT hepática. Respecto al colesterol, el absorbido desde la luz intestinal, parece ser el sustrato preferente para la ACAT, aunque el colesterol sintetizado de novo en la célula epitelial puede también ser utilizado como sustrato (45). Los ácidos biliares actúan como inhibidores de dicha enzima. Presenta un ritmo circadiano paralelo al de la reductasa intestinal, pero de menor amplitud.

Hasta nuestros días se ha podido detectar actividad de esta enzima en el intestino de rata, conejo y hombre (38). En la rata, está presente en alta proporción en yeyuno, disminuyendo a medida que nos desplazamos al ciego. Esta misma distribución la presenta el intestino de conejo y hombre. La localización de la enzima varía también a lo largo del eje cripta-villus, presentándose la mayor cantidad en la zona de la vellosidad (46) (47). Por tanto, la mayor esterificación del colesterol se realiza en aquellas zonas donde mayoritariamente se produce su absorción. No obstante, algunos autores presentan dudas sobre el papel que realiza la ACAT en la esterificación del colesterol absorbido desde el lumen (48). Al mismo tiempo, parece existir una relación inversa entre la actividad biosintética de colesterol (baja en yeyuno y células de las vellosidades) y la esterificante (alta en yeyuno y villus) (49).

Se ha comprobado que la actividad ACAT aumenta como consecuencia de la adición de colesterol a la dieta, pero se observó que este aumento no dependía de la síntesis de proteína enzimática (ya que la cicloheximida no lo inhibía), sino que parece ser el resultado de un aumento en la biodisponibilidad de sustrato (38) (49) (50) (51).

Posteriormente, Purdy y Field (52) postularon que la ACAT, independientemente de factores luminales, estaba también regulada al igual que la HMG-CoA reductasa por las lipoproteínas plasmáticas, lo que apoya la idea de que, de manera análoga a la actividad hepática, la actividad ACAT intestinal es, al menos en parte, un reflejo de la disponibilidad de sustrato en las membranas microsomales.

Gallo et al (47) (53), demostraron que los inhibidores de la ACAT no causaban ninguna alteración en la absorción de colesterol, y que, recíprocamente, la disminución de la absorción de colesterol causada por la interrupción de la circulación entero-hepática no estaba acompañada por una disminución de actividad ACAT. De esta forma concluyeron que la ACAT no era la enzima responsable de toda la esterificación del colesterol que se producía tras su absorción intestinal, atribuyéndole un papel bastante importante a la colesterol-esterasa.

\section{Liberación de lipoproteínas por la mucosa intes-} tinal.

El enterocito libera a la linfa lipoproteínas, concretamente quilomicrones. Se ha demostrado que durante el ayuno, la mayoría del colesterol liberado por el enterocito en forma de lipoproteínas procede del hígado. Durante la absorción activa de triglicéridos se registra un aumento en la síntesis de colesteol en el enterocito, pasando éste a formar la cubierta de los quilomicrones. En esta situación, el intestino contribuye en una cuarta parte al colesterol encontrado en linfa. Por lo tanto, el colesterol nuevamente sintetizado en el enterocito es utilizado principalmente por la célula epitelial, y sólo aumenta marcadamente su síntesis cuando se necesita colesterol en exceso para formación de lipoproteínas durante la absorción de grasas (38). De manera análoga, cuando ratas en ayuno son tratadas con colestiramina, la formación de lipoproteínas por la célula intestinal disminuye (54).

\section{COMPOSICION LIPIDICA DE MEMBRANAS}

La estructura aceptada actualmente para las biomembranas es la de una bicapa lipídica, asimétrica, constituída por fosfolípidos, en cuya matriz se encuentra el colesterol y las proteínas de membrana intrínsecas y extrínsecas. La razón proteínas/lípidos varía dependiendo del tipo de membrana (membrana mitocondrial un $76 \%$, membrana plasmática un $50 \%$ ). 
Todos los fosfolípidos de membrana (fosfatidilcolina, fosfatidilserina, fosfatidiletanolamina, fosfatidilinositol), menos las cardiolipinas, contienen ácidos grasos de 16 a 22 átomos de carbono. Estos ácidos grasos son en su mayoría insaturados, presentando de 1 a 4 dobles enlaces. Todos con conformación cis, lo que permite una mayor compactación. Dependiendo del $\mathrm{pH}$, la cadena polar puede ser neutra (fosfatidilcolina, fosfatidiletanolamina) o negativa (fosfatidilserina, fosfatidilinositol).

Las membranas pueden encontrarse en estado de gel o fluido, dependiendo de la temperatura (55). Los lípidos con ácidos grasos cortos 0 insaturados tienen temperatura de transición más baja y, una forma de adaptación a mayores temperaturas es el aumento en la proporción de ácidos saturados, lo que mantiene el estado de fluidez a temperaturas más altas (56).

El colesterol es un componente de las membranas igualmente determinante de su fluidez. Normalmente, este colesterol se encuentra en forma libre con sólo trazas de colesterol esterificado (55). Por su hidrofobicidad no forma estructuras compactas con él mismo, intercalándose con los fosfolípidos. Su grupo -OH interacciona con la cabeza polar de los fosfo- lípidos, mientras que el anillo esteroideo interacciona con las cadenas de ácidos grasos tendiendo a inmovilizarlos (56). El efecto neto del colesterol sobre la fluidez de membrana depende de la composición lipídica. Debido a las concentraciones altas de colesterol encontradas en eucariotas, el colesterol tiende a hacer la membrana menos fluida a temperaturas ' alrededor de $37^{\circ} \mathrm{C}$.

Las modificaciones en la composición lipídica, producen cambios en la fluidez de membrana, que podrían ser suficientes para alterar diversos procesos celulares tales como: transportes mediados, propiedades de enzimas de membrana y afinidades de hormonas por sus receptores. Pero, los efectos que producen las modificaciones en los lípidos de membrana son muy complejos, varian de unas células a otras y son diferentes en cada uno de los fenómenos estudiados (55).

En los animales existen varias familias de ácidos poliénicos, que difieren entre sí por el número de átomos de carbono existente entre el grupo metilo terminal y el doble enlace más próximo. Estas familias (ver Esquema 4) reciben el nombre de su ácido graso precursor, concretamente, palmitoleico (n-7), oleico (n-9), linoleico (n-6) y linolénico $(n-3)(57)(58)$.

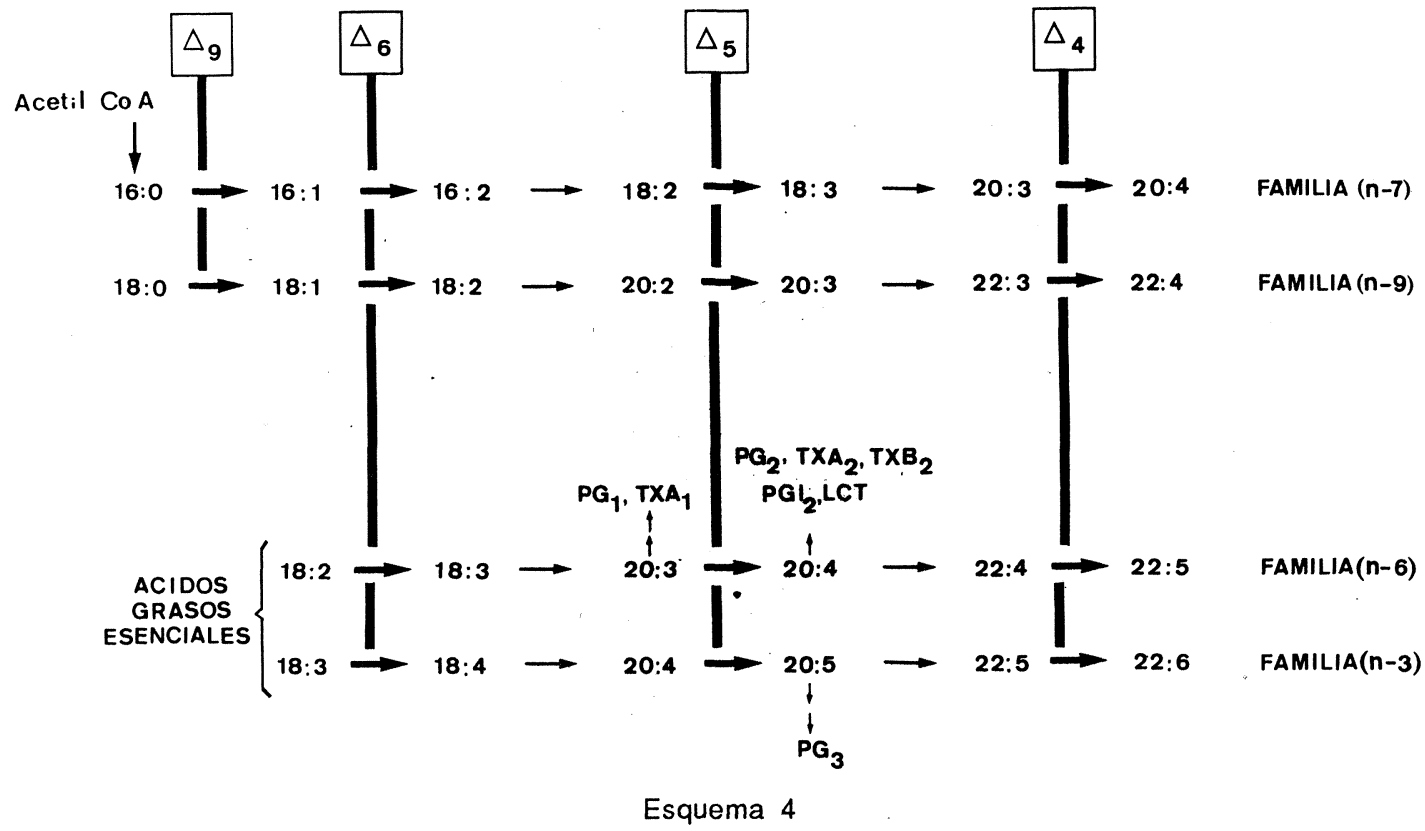

Biosíntesis de ácidos grasos saturados e insaturados y destino de los ácidos poliénicos en los animales. Enzimas desaturasasa $\left(\Delta_{9}, \Delta_{6}, \Delta_{5}\right.$, y $\left.\Delta_{4}\right): \rightarrow$; enzimas elongasas: $\rightarrow$ TX: trombosanos: PG: protaglandinas; PGl: prostaciclinas; LCT: leucotrienos. Familia ( $n-7)$ : 16:0, ácido palmítico; $16: 1$ ( $n-7)$, ácido palmitoleico.

Familia (n-9): 18:0, ácido esteárico; 18:1 (n-9), ácido oleico; 20:3 (n-9), ácido 5, 8, 11-eicosatrienoico.

Familia (n-6): 18:2(n-6), ácido linoleico; $20: 4$ (n-6), ácido araquidónico.

Familia (n-3): 18:3(n-3), ácido $\alpha$-linolénico.

Los ácidos grasos que constituyen los fosfolípidos son sintetizados en el retículo endoplásmico de la célula, a partir de ácido linoleico y -linolénico (ácidos grasos esenciales), que mediante reacciones, alterna- das, de desaturación y elongación dan lugar a ácidos grasos más insaturados y de mayor peso molecular.

De las dos reacciones microsómicas, desaturación y elongación, las de desaturación desempeñan un 
papel primordial en la biosíntesis de ácidos poliénicos y están catalizadas por distintas desaturasas. Brenner (57) (58) (59) ha descrito la $\Delta_{9}, \Delta_{6}, \Delta_{5}$-desaturasas hepáticas, y se postula la existencia de una $\Delta_{4}$-desaturasa. Todas estas enzimas utilizan como sustrato un ácido graso unido a CoA. En general las desaturasas muestran mayor afinidad por el sustrato más insaturado, compitiendo los distintos sustratos por el enzima. El orden de preferencia sería: familia del linolenico>linoleico>oleico>palmitoleico.

La $\Delta_{6}$-desaturasa ocupa una posición clave en la ruta biosintética de los ácidos grasos poliinsaturados, ya que, regula la síntesis de ácido araquidónico (20:4, $n-6)$, catalizando la conversión de ácido linoleico (18:2, $n-6)$ a ácido -linolénico (18:3, n-6). De modo que una modificación de su actividad, repercutiría rápidamente en la biosíntesis de todos los ácidos grasos superiores (57). Los estudios de Brenner et al. (57) (58) (59) (60) han demostrado que la $\Delta_{6}$-desaturasa está bajo control de diferentes factores alimenticios, hormonales y ambientales. La falta de ácidos grasos esenciales aumenta su actividad y los poliinsaturados de cadena larga la disminuyen por un proceso de retroinhibición.

La $\Delta_{5}$-desaturasa cumple una función importante en la desaturación de ácidos grasos de 20 átomos de carbono, principalmente en la conversión del ácido 8 , 11, 14-eicosatrienoico $(20: 3, n-6)$ a $5,8,11,14-$ eicosatetraenoico $(20: 4, n-6)$, ambos precursores de los prostanoides. La actividad $\Delta_{5}$-desaturasa se modifica por componentes alimenticios y hormonales, y mecanismos competitivos y de inducción. Por ejemplo, la deficiencia en ácidos grasos esenciales disminuye su. actividad. La trioleína y los ácidos grasos poliinsaturados de la serie del linoleico producen una reactivación del enzima (60).

Recientemente, Garg et al., (61) han puesto de manifiesto que el enterocito posee actividad $\Delta_{9}$ y $\Delta_{6}$ desaturasa, postulando que el propio enterocito podría sintetizar parte de los ácidos grasos necesarios para formar los fosfolípidos de sus membranas, y para la síntesis de lipoproteínas.

Según lo indicado anteriormente, las desaturasas desempeñan un papel importante en la provisión de estructuras adecuadas para determinar las propiedades fisico-químicas convenientes de las membranas biológicas, y servir, además, de precursores de sustancias específicas de gran actividad biológica. Por ello, en situaciones patológicas como diabetes mellitus, ingestión crónica de alcohol o resección intestinal, las alteraciones en la composición química de las membranas celulares podrían deberse a cambios en la actividad de estas desaturasas, lo que daría lugar a variaciones en las propiedades fisicoquímicas de las membranas y como consecuencia, en las características de los distintos sistemas de transporte.

\begin{abstract}
Modificaciones de la composición lipídica de membranas microsomales y actividad enzimática.
\end{abstract}

Los microsomas se definen como fragmentos de retículo endoplásmico obtenidos mediante la homogeneización de las células de un tejido. Estos fragmentos forman vesículas que se pueden aislar por centrifugación diferencial. La composición media de las membranas microsomales es de un $70-75 \%$ de proteínas y alrededor de un $27 \%$ de lípidos, siendo el $80 \%$ de estos últimos fosfolípidos y el resto colesterol libre (15).

Numerosos trabajos han estudiado la relación entre la composición lipídica de las membranas microsomales y la actividad de las enzimas implicadas en el metabolismo de dichos lípidos.

Spector et al. (18) comprobaron que la actividad ACAT en microsomas hepáticos de ratas alimentadas con una dieta rica en ácidos grasos poliinsaturados era más alta que en los de ratas alimentadas con dieta suplementada con ácidos grasos saturados. A su vez, los microsomas de animales con una dieta rica en poliinsaturados presentaban una mayor proporción en ácidos grasos insaturados, 18:2(n-6) y $20: 4(n-6)$, no registrándose diferencias en el contenido de colesterol o fosfolípidos, postulando que los cambios en la actividad ACAT se debían a una alteración en la composición de ácidos grasos de la membrana.

Field y Salome (50) encontraron resultados análogos en microsomas de intestino de conejo, administrando dietas de distinto contenido en ácidos grasos y colesterol. Field et al., 1987 llegaron a la misma conclusión al estudiar la actividad HMG-CoA reductasa en hígado de conejos. Sin embargo, no descartaron la posibilidad de que el colesterol libre endógeno ejerciera una función reguladora sobre la enzima ACAT, ya que conejos alimentados con colestiramina, mostraron una disminución en los niveles de colesterol microsomal que iba acompañada de un decremento de la actividad ACAT.

Innis (20) observó en ratas controles, una clara relación entre los niveles de $(n-6)$ y $(n-3)$ de microsomas hepáticos y altas actividades ACAT.

Davis y Poznansky (62) encontraron que un aumento en la relación colesterol/fosfolípidos en microsomas de fibroblastos humanos daba lugar a una supresión de la actividad HMG-CoA reductasa y a una disminución de la fluidez de membrana, sugiriendo que el colesterol podría regular su propia biosíntesis, al menos en parte, mediante cambios en la fluidez de membrana producidos por el propio colesterol.

Todas estas observaciones indican que, con independencia de la regulación por el colesterol, la actividad de estas enzimas (HMG-CoA reductasa y ACAT) se afecta por la composición lipídica de la membrana. 


\section{REFERENCIAS}

1. Brown, M.S.; Goldstein, M.-"General scheme for regulation of cholesterol metabolism in mammalian cells. En: Disturbances in Lipid and Lipoproteins Metabolism". J.M. Dietschy, A.M. Gotto y J.A. Ontko, ed. American Physiological Society, Bethesda MD (1978), 173-180.

2. Gill, J.R.-"Control mechanism in sterol uptake and biosynthesis. En: Sterols and Bile Acids".-1985. H. Danielsson and J. Sjovall (eds) Elsevier Science Publishers B.V. (biomedical división).

3. Kenelly, P.J.; Rodwell, J.W.-"Regulation of 3-hydroxy-3methylglutaryl coenzime $A$ reductase by reversible phosphorilation-dephosphorilation".-J. Lipid Res. 26 (1985) 903914.

4. Shapiro, D.J.; Rodwell, V.W.-"Regulation of 3-hydroxy-3methylglutaryl coenzime $A$ reductase and cholesterol synthesis".-J. Biol. Chem. 246 (1971) 3210-3216.

5. Ramírez, H.; Alejandre, M.J.; Segovia, J.L.; García-Peregrin, E.-"Development of the diurnal, rythm of chick 3hydroxy-3-methylglutaryl-CoA-reductase".-Lipids 17 (1982) 434-436.

6. Mitropoulos, K.A.; Balasubramanian, S.; Venkatesan, S.; Reeves, B.E.A.-"On the mechanisms for the regulation of 3-hydroxy-3-methylglutaryl coenzyme $A$ reductase, of cholesterol $7 \alpha$-hydroxylase and of acyl-coenzyme A: cholesterol acyltransferase by free cholesterol".-Biochim. Biophys. Acta 530 (1978) 99-110.

7. Ramírez, H.; Alejandre, M.J.; Segovia, J.L.; García-Peregrin, E.-"Different thermal behavior of neonatal hepatic and cerebral 3-hydroxy-3-methylglutaryl-CoA-reductase ".-Lipids 16 (1978) 552-554.

8. Sabine, J.R.; James, M.J.-"The intracellular mechanism responsible for dietary back control of cholesterol synthesis".-Life Sci. 18 (1978) 1185-1192.

9. Rodwell, V.M.; MC Namara, D.J.; Shapiro, D.J.-"Regulation of hepatic 3-hydroxy-3-methylglutaryl coenzime A reductasen.-Adv. Enzymol, 38 (1973) 373-412.

10 Brown, M.S.; Goldstein, J.L.; Dietschy, J.M.--"Active and inactive forms of 3-hidroxy-3-methylglutryl coenzime A reductase in the liver of the rat. Comparision with rate of cholesterol synthesis in different physiological states".-J. Biol. Chem. 254 (12) (1979) 5144-5149.

11. Packard, C.J.Shepferd, J.-“-"The hepatobiliary axis and lipoprotein metabolism: effects of bile acid sequestrants and ileal bypass surgeryn.-J. Lipid Res. 23 (1982) 1081-1098.

12. Haugen, R.; Norum, K.R.-"Coenzyme A-dependent esteri fication of cholesterol by rat intestinal mucosa".-Scand. J. Gastroenterol. 11 (1076).

13. Balasubramaniam, S.; Venkatesen, S.; Mitropoulos, K.; Peters, T.J.-"The submicrosomal localization of acyl-coenzyme A-cholesterol acyltransferase and its substrate, and of cholesteryl esters in rat liver".-Biochem., J. 174 (1976) 863872.

14. Shefer, S.; Cheng, F.W.; Hanser, S.; Batta, A.T.; Salen, G.-"Regulation of bile acid synthesis, Measurement of cholesterol $7 x$-hydroxylase activity in rat liver microsomal preparations in the absence of endogenous cholesterol". $-J$. Lipid Res. 22 (1981) 532-536.

15. Ericksson, L.C.; Depierre, J.W.; Dallner, G.-"Preparations and properties of microsomal fractions. En Hepatic cytocrome P-450 monoxygenase system".-J.B. Shenkman y D. Kupfer (ed). Pergamon Press (1980).
16. Jones, A.; Glomset, J.-“Biosinthesis function and metabolism of sterol esters. En: Sterols and Bile Acids. $-H$ Danielsson and J. Sjovall (eds), Elsevier Science Publishers B.V. (Biomedical División) (1985).

17. Spector, A.A.; Yorek, M.A.-"Membrane lipid composition and cellular function.-J. Lipid Res. 26 (1985) 1015-1035.

18. Spector, A.A.; Kaduce, T.L.; Dane, R.W.-“Effect of dietary fat saturation on acylcoenzime A: cholesterol acyltransferase activity of rat liver microsomes". J. Lipid Res. 21 (1985) 169-179.

19. Mathur, S.N.; Armstrong, M.L.; Alber, C.A.; Spector, A.A.-"Hepatic acylcoenzyme A: cholesterol acyltransferase activity during diet-induced hypercholesterolemia in cynomolgus monkeys".-J. Lipid Res. 22 (1981) 659-667.

20. Innis, S.-"The activity of 3-hydroxy-3-methylglutaryl-Co A reductase and acyl-Co $A$ : cholesterol acyltransferase in hepatic microsomes from males, females and pregnant rats. The effect of cholestyramine treatment and the relationship of the enzyme activity to microsomal lipid composition ${ }^{n}$.-Biochim. Biophys. Acta 875 (1986) 355-361.

21. Gavey, K.L.; Noland, B.J.; Scallen, T.J.-"The participation of sterol carrier protein in conversion of cholesterol to cholesterol ester by rat liver microsomes".-J. Biol. Chem. 256 (1981) 2993-2999.

22. Myant, N.B.; Mitropoulos, K.A.-"Cholesterol 7xhydroxylase".-J. Lipid Res. 18 (1977) 135-153.

23. Ogura, M.; Shiga, J.; Yamasaki, K.-"Studies on the cholesterol pool as the precursor of bile acids in the rats" $-J$. Biochem. (1971) 70-967.

24. Drevon, C.A.; Engelhorn, S.C.; Steinberg, D.-"Secretion of very low density proteins enriched in cholesterol esters by cultured rat hepatocytes during stimulation of intracellular cholesterol esterificationn ${ }^{n}$-J. Lipid Res. 21 (1980) 1065 1071.

25. Siperstein, M.D.-"The homeostatic control of cholesterol synthesis in liver".-Am. J. Clin. Nutr. 8 (1960) 645-650.

26. Westergaard, H.; Dietschy, J.M.-"Delineation of dimensions and permeability characteristics of the two major difussion barriers to passive mucosal uptake in rabbit intestine ${ }^{n} .-J$. Clin. Invest. 54 (1976) 718-732.

27. Thomson, A.B.R.; Dietschy, J.M.-"Intestinal lipid absorption: major extracellular and intracellular events".-En: Physiology of the Gastrointestinal Tract, L.R. Jonhoson (ed) Raven Press, New York.

28. Gallo-Torres, H.E.; Miller, O.N.; Hamilton, J.G.-"Further studies in the role of bile salts in cholesterol esterification and absorption from the gut ${ }^{n}$.-Arch. Biochem. Biophys. 143 (1971) 22-36.

29. Thomson, A.B.R.; Hotke, C.A.; O'Brien, B.D.; Weinstein W.M.-"Intestinal uptake of fatty acids and cholesterol in four animals species and man: role of unstirred water layer and bile salt micellen.-Comp. Biochem. Physiol. 75A (2) (1983) 221-232.

30. Thomson, A.B.R.-"Aging and cholesterol uptake in the rabbit jejunum. Role of the bile salt micelle and the unstirred water layer".-Dig. Dis. Science 26 (10) (1981) 890-896.

31. Hollander, D.; Morgan, D.-"Increase in cholesterol intestinal absorption with aging in the rat".-Exp. Geront. 14 (1979) 201-204.

32. Thomson, A.B.R.-"Influence of sites and unstirred layers on the unidirectional flux of cholesterol and fatty acids into the rabbit intestine".-J. Lipid Res. 21 (1980) 1097-1107.

33. Field, F.J.; Erickson, S.K.; Shrewsbury, M.A.; Cooper, A,D.-"3-Hydroxy-3-methylglutaryl coenzime A reductase from rat intestine: subcellular localization and in vitro regulation".-J. Lipid Res. 23 (1982) 105-113. 
34. Thomson, A.B.R.; Keelan, M.; Clandinin, M.T.-“"Onset and persistence of changes in intestinal transport following dietary fat manipulation".-Lipids 22 (1987) 22-27.

35. Thomson, A.B.R.; Keelan, M. Clandinin, M.T.; Walker, K.-"Dietary fat selectivity alters transport properties of rat jejunum".-J. Clin. Invest. 77 (1986) 279-298.

36. Turley, S.D.; Andersen, J.M.; Dietschy, M.-"Rates of sterol synthesis and uptake in the major organs of the rat in vivo".-J. Lipid Res. 22 (1981) 551-569.

37. Spady, D.K.; Dietschy, J.M.-"Sterol synthesis in vivo in 18 tissues of the squirrel monkey, guinea pig, rabbit, hamster and rat".-J. Lipid Res, 24 (1983) 303-315.

38. Stange, E.F.; Dietschy, J.M.-"Cholesterol absorption and metabolism by the intestinal epitheliumn ${ }^{n}$-(1985) En: Sterols and Bile Acids. Elsevier Science Publishers B.V. (biomedical Division).

39. Panini, S.R.; Lehrer, G.; Rogers, $H_{\text {,; }}$ Rudney, H.-“Distribution of 3-hydroxy-3-methylglutaryl coenzime $A$ reductase and alkaline phosphatase activities in isolated ileal epithelial cells of fed, fasted, cholestyramine-fed, and 4-aminopyrazolo[3, 4, d-jpyrimidinetreated rats ${ }^{n}$.-J. Lipid Res. 20 (1979) 879889.

40. Panini, S.R.; Rudney, H.-"Short term reversible modulation of 3-hydroxy-3-methylglutaryl coenzime $A$ reductase activity in isolated epithelial cells from rat ileum. ${ }^{n} .-\mathrm{J}$. Biol. Chem. 255 (1980) 11633-11636.

41. Einarsson, K.A.; Hellstrom, K.; Kallner, M.-"Feed-back regulation of bile acid synthesis in man".-Metabolism 22 (1973) 1477-1479.

42. Stange, E.F.; Suckling, K.E.; Dietschy, J.M.-"Synthesis and coenzime A-dependent esterification of cholesterol in rat intestinal epithelium, differences in cellular localization and mechanisms of regulation".-J. Biol. Chem. 258 (1983) 1286812875.

43. Anderson, J.M.; Dietschy, J.M.--"Regulation of sterol synthesis in 15 tissues of rat. II. Role of rat and human high and low density plasma lipoproteins and of rat chylomicron remnants".-J. Biol. Chem. 252 (1977) 3652-3659.

44. Field, F.J.; Albright, E.J.; Mathur, S.N.-"Effect of dietary n3 fatty acids on HMG-COA reductase and ACAT activities in liver and intestine of the rabbit" ${ }^{n}$.-J. Lipid Res. 28 (1987) 56-58.

45. Bennet-Clarck, S.B.; Tercyak, A.-"Reduced cholesterol transmucosal transport in rats inhibited mucosal acyl CoAcholesterol acyltransferase and normal pancreatic functionn.-J. Lipid Res. 25 (1984) 148-159.

46. Field, F.J.; Albright, E.J.; Mathur, S.N.-"The effect of hypothyroidism and thyroxine replacement on hepatic and intestinal HMG-COA reductase and ACAT activities and biliary lipids in the rat".-Metabolism 35 (12) (1986) 1085-1089.

47. Gallo, L.L.; Myers, S.; Vahouny, G.V.-"Rat intestinal Acyl Coenzime A: cholesterol Acyl Transferase properties and localization".-Proc. Soc. Ex. Biol. Med. 177 (1984) 188196.

48. Gallo, L.L.; Bennet-Clark, S.; Myers, S.; Vahouny, G.V.-"Colesterol absorption in rat intestine: role of cholesterol esterase and acyl coenzime A: cholesterol acyl transferase".-J. Lipid Res. 25 (1984) 604-612.
49. Field, F.J.; Erickson, S.K.; Cooper, A.D.-“'Intestinal acylcoenzyme A: cholesterol acyltransferase (ACAT). Regulation in vivo and in vitro".-Gastroenterology 83 (1982) 873 880.

50. Field, F.J.; Salome, R.G.-"Effect of dietary fat saturation, cholesterol and cholestiramine on acyl-CoA: cholesterol acyltransferase activity in rabbit intestinal microsomes".-Biochim. Biophys. Acta 712 (1982) 557-570.

51. Helgerud, P.; Haugen, R.; Norum, K.R.-"The effect of feeding and fasting on the aactivity of acyl-CoA: cholesterol acyltransferase in rat small intestine".-Eur. J. Clin. Invest. 12 (1982) 493-500.

52. Purdy, B.H.; Field, F.J.-"Regulation of acylcoenzime A: cholesterol acyltransferase and 3-hydroxy-3-methylglutaryl coenzime $A$ reductase activity by lipoproteins in the intestine of parabionts rats".-J. Clin Invest. 74 (1988) 351-357, 1984.

53. Gallo, L.L.; Wadsworth, J.A.; Vahouny, G.V.-“Normal cholesterol absorption in rats deficient in intestinal acyl coenzime A: cholesterol acyltransferase activity".-J. Lipid Res. 28 (1987) 381-387.

54. Gaugl, A.; Ockner, R.K.-“'Intestinal metabolism of lipids and lipoproteins".-Gastroenterology 68 (1975) 167-189.

55. Chapman, D.; Kramers, M.T.C.; Restall, C.J.-“Cholesterol and biomembranes structures".-En: Sterols and Bile Acids. H. Danielsson and Sojvall J. (Eds.) Elsevier Publishers B.V. (1985).

56. Darnell, J.; Lodish, H.; Baltimore, D.-“Molecular Cell Biology".-Capítulos 5 y 14. Scientific American Books (Eds) (1986).

57. Brenner, R.R.-"Regulatory function of $\Delta 6$-desaturase Key enzime of polyunsatured fatty acid synthesis".-En: Bazan, N.G.; Brenner, R.R., y Giusto, N.M.-Function and biosynthesis of lipids.-Adv. in Exp. Med. and Biol., vol. 83, 8599. Plenum Press. New York. (1977).

58. Brenner, R.R.- "The oxidative desaturation of unsaturated fatty acids in animals".-Mol. Cell. Biohem. 3 (1974) 41-52.

59. Brenner, R.R.; Peluffo, R.O.-"Effect of saturated and unsaturated fatty acids on the desaturation in vitro of palmitic, stearic, oleic, linoleic and linolenic acids".-J. Biol. Chem. 241 (1966) 5213-5219.

60. Brenner, R.R.-“Nutritional and hormonal factors influencing desaturation of essential fatty acids".-En Holman, R.T. (Ed): Essential fatty acids and prostaglandins.-Progress in Lipid Res., vol XX, 41-47, Pergamon Press, Nueva York. (1981).

61. Garg, M.L.; Keelan, M.; Thomson, A.B.R.; Clandinin, M.T.-"Fatty acid desaturation in intestinal mucosan"-Biochim. Biophys. Acta 958 (1988) 139-141.

62. Davis, P.J.; Poznansky, M.J.-"Modulation of 3-hydroxy-3methylglutaryl CoA reductase by changes in microsomal cholesterol content or phospholipid composition".-Proc. Nat. Acad. Sci. USA 84 (1987) 118-121.

(Recibido: Octubre 1990) 